\title{
DÜBLIN
}

Technological University Dublin

ARROW@TU Dublin

\section{A Comparative Study of Chisel for FPGA Design}

\author{
Paul Lennon \\ Technological University Dublin, paul.lennon@outlook.com \\ Richard Gahan \\ Technological University Dublin, richard.gahan@it-tallaght.ie
}

Follow this and additional works at: https://arrow.tudublin.ie/ittengcon

Part of the Electrical and Computer Engineering Commons

\section{Recommended Citation}

P. Lennon and R. Gahan, "A Comparative Study of Chisel for FPGA Design," 2018 29th Irish Signals and Systems Conference (ISSC), 2018, pp. 1-6, doi: 10.1109/ISSC.2018.8585292.

This Conference Paper is brought to you for free and open access by the School of Engineering at ARROW@TU Dublin. It has been accepted for inclusion in Conference Papers by an authorized administrator of ARROW@TU Dublin. For more information, please contact arrow.admin@tudublin.ie, aisling.coyne@tudublin.ie, gerard.connolly@tudublin.ie.

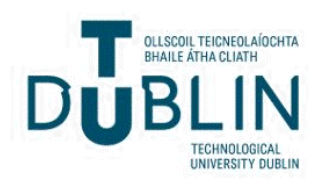




\title{
A Comparative Study of Chisel for FPGA Design
}

\author{
Paul Lennon*, Richard Gahan ${ }^{\dagger}$ \\ Department of Electronic Engineering, Institute of Technology Tallaght \\ Email: *paul.lennon@outlook.com, ${ }^{\dagger}$ richard.gahan@it-tallaght.ie
}

\begin{abstract}
This paper presents the results of a comparative study conducted into designing with the Chisel hardware construction language against the Verilog hardware description language across a range of standard-library and bespoke FPGA design components including an N-bit FIFO, a round-robin arbiter and a complex, scalable arbiter. Comparison metrics such as maximum operating frequency, silicon area, design flow run-time, source-code density and maintainability, simulation run-time and speed of coding are employed to evaluate the merits of designing with Chisel. Each component is implemented with a deep lowlevel hardware understanding with an aim to evaluate the merits of designing with Chisel from a hardware designers' perspective. The authors discover Chisel's merits for realising synthesizable repetitive designs such as in $\mathrm{SoC}$ development, experiencing the benefits of Chisel's object-oriented background in enhancing code maintainability and scalability, and implementation efficiency. However, the authors foresee that Chisel will compliment rather than replace traditional HDLs for RTL design applications due to its limitations in terms of behavioural modelling.
\end{abstract}

Index Terms-Chisel, High-Level Synthesis.

\section{INTRODUCTION}

A S FPGA designs increase in size to millions of gates, the industry requires a more efficient approach to Digital Systems design compared with traditional HDL techniques to increase developer productivity by reducing the complexity in realizing hardware designs whilst reducing run-time in the design flow. To reduce FPGA design complexity, it is expected that the design space abstraction level will be raised from Register-Transfer Level (RTL) to a high-level, object-oriented software perspective. Chisel presents a possible solution to this requirement.

Chisel is a hardware construction language (HCL), designed in UC Berkeley that supports advanced hardware design using highly parameterized generators and layered domain-specific languages[1]. In short, Chisel describes code generators which generate Verilog suited to processing using industry standard RTL design flows. Chisel is implemented as a package of Scala class libraries, interpreted by the Java Virtual Machine (JVM). The conceptual design of the Chisel HCL is to address the lack of Object-Oriented Programming (OOP) and preprocessor constructs in existing HDLs to provision for the construction of parameterize-able code generators.

Traditional approaches to complex, repetitive FPGA designs implement custom scripts in high-level scripting languages to auto-generate Verilog on a per module basis but require the implementation of the entire design to span multiple languages

978-1-5386-6046-1/18/\$31.00 (c)2018 IEEE as the core design remains as hand-coded Verilog. High-level scripting languages such as TCL, Perl and Python are used to implement Verilog generators of this style. Chisel offers this high-level functionality intrinsically whilst the entire design can be expressed in a single language. A documented advantage of Chisel is its ability to perform rapid simulation in a $\mathrm{C}++$ simulation environment in comparison to the performance of industry standard Verilog pre-synthesis simulation environments[1]. According to Waterman et al, an $8 \mathrm{x}$ performance increase in simulation run-time was experienced for a 64-bit 5 stage RISC processor.

Section II of this document provides an overview of Chisel. Sections III - V describe the comparison performed on standard-library design components implemented by the authors, while Section VI and Section VII describe the use of Chisel for simulation and the caveats encountered during the comparison study respectively. Section VIII concludes the authors' comparison of Chisel.

\section{CHISEL}

Chisel is implemented as a distributable Scala library. Scala is a combination of an object-oriented and functional programming language interpreted by the Java Virtual Machine.

Chisel has a small but growing number of adopters, with a large code base containing standard-library, pre-canned RTL design components in place. In addition, the RISC-V Foundation's open-source RISC-V processor project is available as a large scale, active design project for inspection along with that of other leading RISC based processor research groups. A limited number of tutorials are provided to get developers off the ground with Chisel. Licensing of the Chisel library is governed via a modified BSD license.

While dedicated Chisel support is not provided by tool vendors, Scala based interpreters can be utilized for Chisel development which are available across all platforms. The Scala plugin for Eclipse was used by the authors for Chisel development. The Chisel library (Scala library implementing Chisel API) is included in the Scala project using SBT, a standalone Scala build utility with an Eclipse plugin enabled, to simplify linking to the Chisel library. Once the Scala project is compiled, a Verilog file is generated which can then be processed with any standard RTL design flow.

\section{ROUND-ROBIN ARBITER}

A standard 4-input state-machine based round-robin arbiter as shown in Figure 1 was used as a benchmark component 
to compare Chisel-generated Verilog and hand-coded Verilog designs. The round-robin arbiter implementation supports an "ack" input to allow masters to extend the granted control for multi-cycle operations on shared resources. This block was

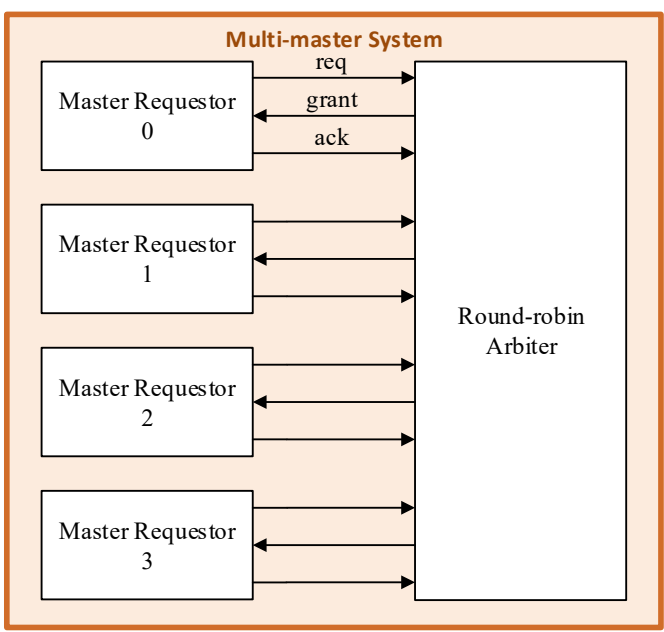

Fig. 1. Multi-master System Arbiter block diagram.

chosen for analysis due to its widespread usage in digital design whilst its standard sequential state-machine and combinatorial elements are sufficient to verify Chisel's theoretical functionality. Effort was placed upon achieving functional equivalence between the two implementations.

\section{A. Functional Equivalence}

Verification of functional equivalence was performed across 10 million randomly generated test vectors in the testbench platform illustrated in Figure 2.

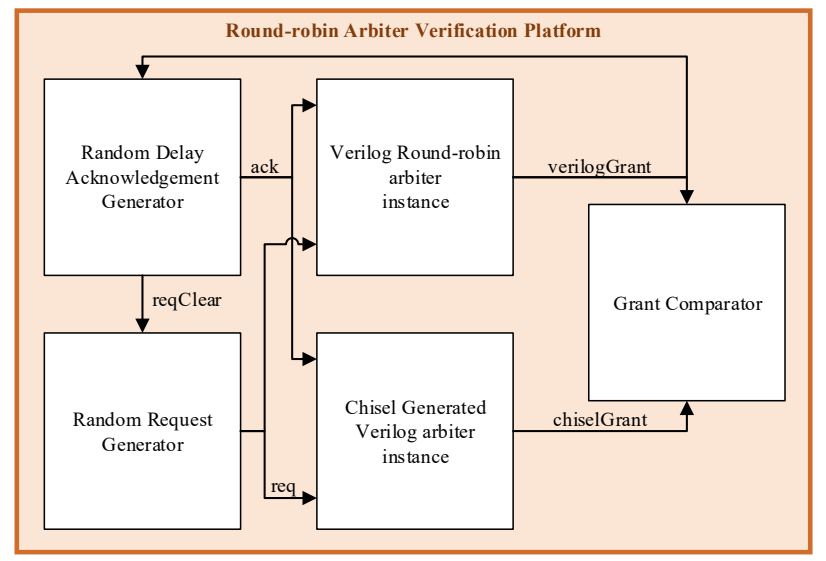

Fig. 2. Round-robin Arbiter Functional Verification platform.

\section{B. Results}

1) Code density and maintainability: The Scala implementation spans 220 lines compared to 260 lines for the
Verilog equivalent. Originally the arbiter was implemented with Chisel 2 before porting the design to Chisel3. The lack of a default clause in Chisel's switch statement forced the design to use a when, elsewhen, otherwise clause to handle combinatorial outputs from the state-machine in the same construct as the sequential state assignments.

2) Design Flow run-time: The design flow run-time was measured from Synthesis through to Static Timing Analysis, with no significant differences uncovered. Two different families of Microsemi FPGAs were chosen as the target hardware as they are supported by separate build tools which provided a wider platform for analysis, helping to identify Verilog interpretation variances in the results.

TABLE I

ROUND-ROBIN ARBITER DESIGN Flow RUN-TIME

\begin{tabular}{|c|c|c|}
\hline Device & HDL & Time (M:S) \\
\hline Microsemi SmartFusion2 & Chisel & $02: 07$ \\
\hline Microsemi SmartFusion2 & Verilog & $01: 59$ \\
\hline Microsemi PolarFire & Chisel & $06: 23$ \\
\hline Microsemi PolarFire & Verilog & $06: 08$ \\
\hline
\end{tabular}

3) Silicon Area: The results show comparable resource logic element utilization for all devices apart from SmartFusion 2 where additional logic gets consumed by the hand-coded implementation.

TABLE II

ROUND-ROBIN ARBITER RESOURCE UTILIZATION

\begin{tabular}{|c|c|c|c|}
\hline Device & HDL & Sequential & Combinatorial \\
\hline Microsemi SmartFusion2 & Chisel & 8 & 45 \\
\hline Microsemi SmartFusion2 & Verilog & 8 & 55 \\
\hline Microsemi PolarFire & Chisel & 9 & 49 \\
\hline Microsemi PolarFire & Verilog & 8 & 50 \\
\hline
\end{tabular}

4) Performance: The maximum operating frequency of both implementations is almost identical for each device analyzed.

Chisel's lack of support of asynchronously reset sequential

TABLE III

Round-Robin ARBITER PERFormance

\begin{tabular}{|c|c|c|}
\hline Device & HDL & Max. Frequency (MHz) \\
\hline Microsemi SmartFusion2 & Chisel & 330 \\
\hline Microsemi SmartFusion2 & Verilog & 321 \\
\hline Microsemi PolarFire & Chisel & 500 \\
\hline Microsemi PolarFire & Verilog & 500 \\
\hline
\end{tabular}

elements impacts on the operating frequency of the Chisel generated Verilog designs. Selecting an asynchronous reset topology in the Verilog implementation boosts the maximum operating frequency by up to $17 \%$ in the case of SmartFu$\operatorname{sion} 2$.

\section{FIFO}

An N-bit FIFO was implemented using Chisel in order to evaluate Chisel's configurability benefits. The FIFO implementation, shown in Figure 3, has a configuration parameter to either infer RAM or to use a custom RAM tiler implemented in Chisel to instantiate an array of device specific RAM blocks. 
The number and pattern in which RAM blocks are tiled is based on the dimensions of the FIFO and the dimensions of the target device's RAM blocks. The Verilog macro for the target device's RAM block is defined in a Verilog file and instantiated as a blackbox component in Chisel.

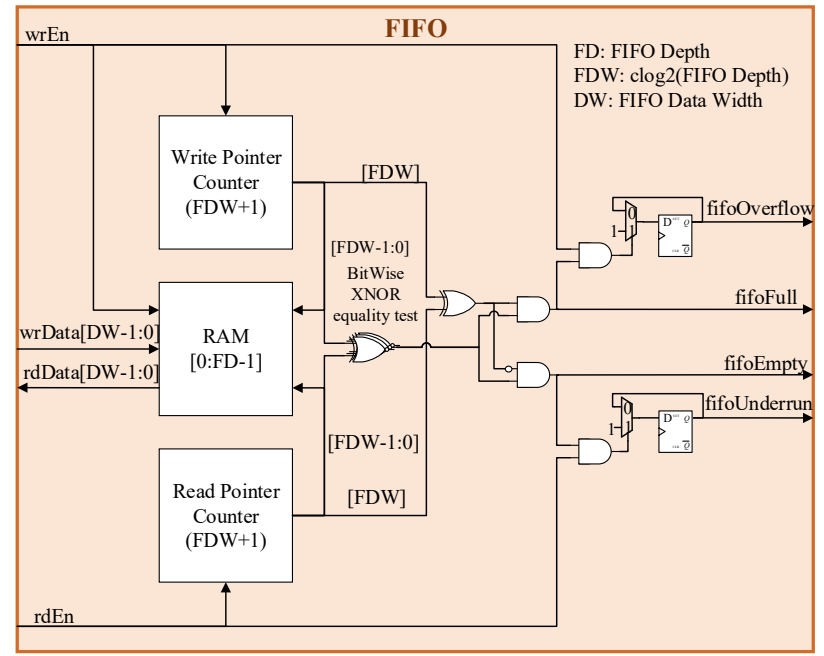

Fig. 3. N-bit FIFO block diagram.

\section{A. Results}

1) Code density and maintainability: The Scala and Verilog FIFO implementations span approximately 90 lines where RAM inference is selected in the Chisel case. The RAM cascader implementation spans a total of 85 lines. Traditionally, RAM cascaders are implemented as a component of the Vendor's tool such as CoreGen in Xilinx Vivado. Integration of blocks produced by Vendor specific RAM generators removes configurability from IP and ties it to a particular Silicon Vendor. Chisel allows for the construction of the RAM cascader within the same source language with the port size and address depth of RAM blocks configurable to allow the tiler to support RAM blocks from any Silicon Vendor.

2) Design Flow Run-time: The design flow run-time was measured from Synthesis through to Static Timing Analysis, with no significant differences uncovered.

TABLE IV

N-BIT FIFO DESIGN FLOW RUN-TIME

\begin{tabular}{|c|c|c|}
\hline Device & HDL & Time (M:S) \\
\hline Microsemi SmartFusion2 & Chisel & $01: 58$ \\
\hline Microsemi SmartFusion2 & Verilog & $01: 59$ \\
\hline Microsemi PolarFire & Chisel & $06: 06$ \\
\hline Microsemi PolarFire & Verilog & $06: 15$ \\
\hline
\end{tabular}

3) Silicon Area: The results show comparable resource utilization for all devices.

4) Performance: The maximum operating frequency of both implementations is identical for each device analyzed.
TABLE V

N-BIT FIFO RESOURCE UTILIZATION

\begin{tabular}{|c|c|c|c|}
\hline Device & HDL & Sequential & Combinatorial \\
\hline Microsemi SmartFusion2 & Chisel & 12 & 34 \\
\hline Microsemi SmartFusion2 & Verilog & 12 & 36 \\
\hline Microsemi PolarFire & Chisel & 12 & 37 \\
\hline Microsemi PolarFire & Verilog & 12 & 38 \\
\hline
\end{tabular}

TABLE VI

N-BIT FIFO PERFORMANCE

\begin{tabular}{|c|c|c|}
\hline Device & HDL & Max. Frequency (MHz) \\
\hline Microsemi SmartFusion2 & Chisel & 250 \\
\hline Microsemi SmartFusion2 & Verilog & 250 \\
\hline Microsemi PolarFire & Chisel & 429 \\
\hline Microsemi PolarFire & Verilog & 429 \\
\hline
\end{tabular}

\section{Scalable Complex Arbiter}

In order to explore the benefits of designing parameterizable code generators using Chisel, a configurable arbiter design was explored. The arbiter shown in Figure 4 allows any number of requestors to be associated with any number of fixed priority levels, with round-robin arbitration performed on requests of the same priority level. As before, an acknowledgement input is provided for each master controller to allow the registered grant output assertion to be extended to support multi-cycle operations on a shared resource. The number of wire mapping possibilities in the prioritization and deprioritization crossbar components dramatically increase based on the number of requestors and the number of priority levels. To reduce complexity, it is a requirement of the design that all enabled priority levels are contiguous, starting at priority 0 , with all enabled priority levels having at least one requestor associated with it. Implementing a configurable crossbar of this nature is not possible in Verilog due to the lack of array-basedprocessing pre-processor constructs. In order to realize this circuit using Verilog, a custom TCL Verilog generator was implemented to determine the wire mapping required in the crossbars. The TCL implementation is somewhat complex using variable substitution to switch between arrays due to TCLs lack of support for two dimensional arrays. Although Perl or Python would have been better suited to this scripting application, TCL was chosen as both the Xilinx Vivado and Microsemi Libero FPGA design environments support an inbuilt TCL interpreter.

The scalable fixed-priority arbiter component shown in Figure 5 was implemented using the combinatorial logic described in [2]. Although unclear from Chisel's documentation, combinatorial logic operations of this type where the value of one bit in a signal is dependent on another bit of the same signal requires use of the Chisel3 Vecs type in place of the standard UInt type.

Two instantiations of this fixed priority arbiter are used to form a configurable N-bit round-robin arbiter as shown in Figure 6. One round-robin arbiter is instantiated per active fixed priority level. Both the fixed priority and round-robin arbiters will pass-through requests if only one requestor is assigned to a given priority level or one priority level is enabled. 


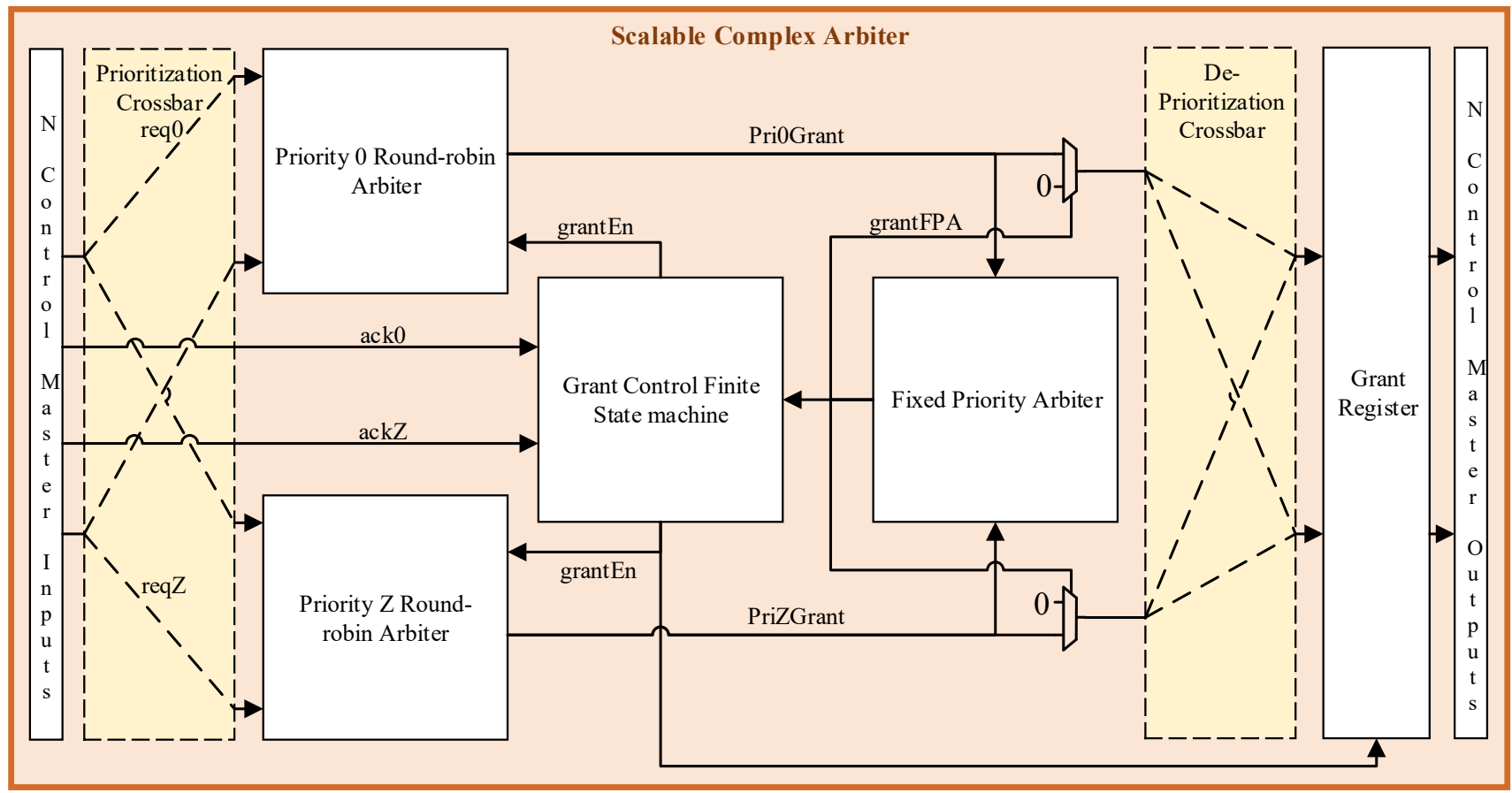

Fig. 4. Scalable Complex Arbiter block diagram.

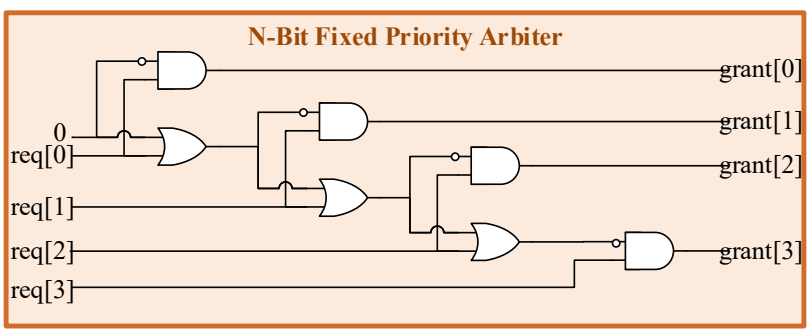

Fig. 5. N-bit Fixed Priority Arbiter block diagram.

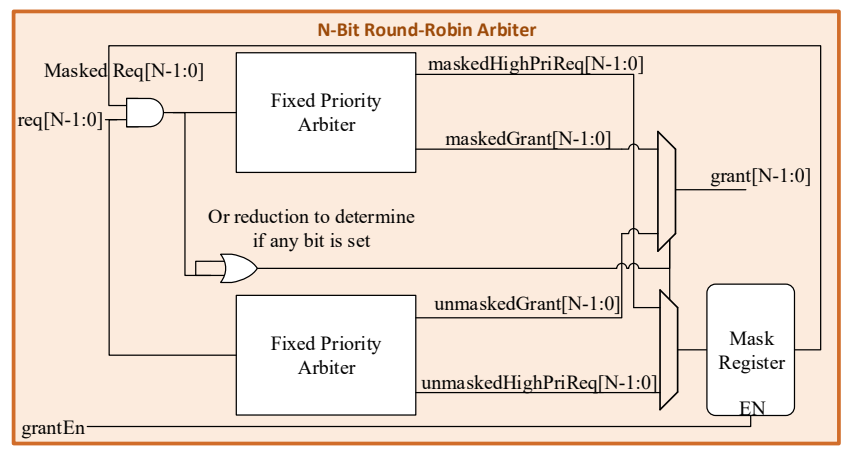

Fig. 6. N-bit Round-robin Arbiter block diagram.

\section{A. Results}

1) Code density and maintainability: The Chisel implementation of the scalable complex arbiter requires 250 lines of Chisel source code whilst the Verilog implementation requires 400 lines, 100 of which are Verilog with the rest accounting for the TCL source required to build the custom Verilog generator. A significant time saving is experienced when implementing this design with Chisel as two-dimensional array processing can be performed to determine the wire mapping requirements in the crossbars. In addition, Chisel's vectorized instantiation can be used to instantiate one round-robin arbiter per priority level. As with most operations in Chisel, there are many possible implementations but the approach taken was to create an immutable array of objects of the roundRobinArbiter class, passing a different "request count" parameter to each instance as each priority level may have a unique number of requests associated with it as shown in the code snippet.

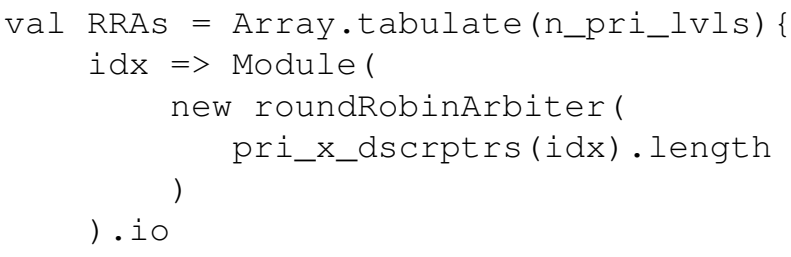

Although vectorized instantiation is permitted in Verilog 2001, the Verilog specification clearly states the behaviour of port mapping leaving parameter mapping open to interpretation by Tool Vendors. In the case of wire mapping, if the signal width being mapped to the lower level port is $\mathrm{N}$ times the width of 
the lower-level port of width $\mathrm{X}$ where the module is being instantiated $\mathrm{N}$ times, bits [X-1:0] are mapped to instance 0 , [2X-1:X] to instance 1 and so forth. In Synplify Pro, the same parameter is mapped to each instance, regardless of the width of the parameter in the higher-level module which prevents the use of vectorized instantiation in Verilog where each instance requires unique parameter mapping.

Scala provides powerful in-built constructs which when coupled with the Chisel library allow complex, repetitive operations to be condensed into fewer lines of source code. Adding to this, Chisel simplifies the implementation of this design as the solution can be realized within a single language, with the ability to pass arrays as arguments to Chisel classes being another key advantage that Chisel possesses over Verilog for aiding design productivity and maintainability.

2) Coding speed and complexity: Given the authors experience at coding with TCL, the implementation time of the TCL based Verilog generator was not hampered by syntactical errors, however, the implementation took $3 \mathrm{x}$ longer than that of the Chisel generator. Composing the architecture of the TCL generator was significantly more difficult compared with the Chisel solution; The design effort went into resolving bugs in the TCL defined generator which was not RTL aware whereas the Chisel implementation generated directed warnings and error messages which helped architecture issues to be detected and isolated with greater efficiency.

3) Design Flow Run-time: The design flow run-time was measured from Synthesis through to Static Timing Analysis, with the hand-coded Verilog implementation outperforming the Chisel generated Verilog solution by $5 \%$ in the case of SmartFusion2 for a 128 requestor, 8 priority configuration.

TABLE VII

Scalable Complex Arbiter Design Flow Run-time

\begin{tabular}{|c|c|c|}
\hline Device & HDL & Time (M:S) \\
\hline Microsemi SmartFusion2 & Chisel & $05: 15$ \\
\hline Microsemi SmartFusion2 & Verilog & $05: 00$ \\
\hline Microsemi PolarFire & Chisel & $08: 52$ \\
\hline Microsemi PolarFire & Verilog & $08: 32$ \\
\hline
\end{tabular}

4) Silicon Area: The results show comparable resource utilization.

TABLE VIII

SCAlable Complex ARbiter Resource Utilization

\begin{tabular}{|c|c|c|c|}
\hline Device & HDL & Sequential & Combinatorial \\
\hline Microsemi SmartFusion2 & Chisel & 273 & 1386 \\
\hline Microsemi SmartFusion2 & Verilog & 263 & 1313 \\
\hline Microsemi PolarFire & Chisel & 263 & 1334 \\
\hline Microsemi PolarFire & Verilog & 263 & 1339 \\
\hline
\end{tabular}

5) Performance: The maximum operating frequency of both implementations is almost identical for each device analyzed.

\section{Vi. Simulation}

The merits of Chisel for rapid $\mathrm{C}++$ simulation reported by Waterman et al. cannot be accredited to Chisel itself,
TABLE IX

Scalable Complex Arbiter Performance

\begin{tabular}{|c|c|c|}
\hline Device & HDL & Max. Frequency (MHz) \\
\hline Microsemi SmartFusion2 & Chisel & 102.69 \\
\hline Microsemi SmartFusion2 & Verilog & 98.57 \\
\hline Microsemi PolarFire & Chisel & 169.70 \\
\hline Microsemi PolarFire & Verilog & 178.80 \\
\hline
\end{tabular}

since the $\mathrm{C}++$ backend flow was deprecated in Chisel3. Instead Verilator, a 3rd party tool for converting Verilog to $\mathrm{C}++$ models is responsible for this productivity boost. This move away from a $\mathrm{C}++$ backend output from FIRRTL was conducted to allow co-simulation of Chisel generated Verilog and black-box, hand-coded Verilog modules, something which hampered the simulation of Heilmann et al.'s implementation of the Heston Model[3]. In this instance, the Chisel-generated Verilog is passed to Verilator which creates $\mathrm{C}++$ simulation models of the Verilog design. However, this can be performed with any Verilog source. The built-in FIRRTL peek-poke tester provides a means to rapidly perform command-line basic functionality testing by developers prior to the design being handed-off for full Verification. It's not anticipated that Chisel will replace SystemVerilog for the construction of the Verification environment. As with all high-level languages used for RTL simulation, the generic naming of internal signals below top-level ports in the design make the internals of the design difficult to explore as signal names are not logical and indicative of the signals functionality.

\section{CAVEATS}

Significant changes were made to the Chisel API between Chisel2 and Chisel3, making it difficult to migrate existing designs to the latest release. Whilst a porting guide is provided, changes to the interface to the backend flow for generating Verilog are undocumented. Also, certain constructs such as the switch statement are moved to the chisel3.utils library from the chisel3 core library. However, Chisel3 provides a more structured API to Chisel 2 and it is anticipated that a wholesale change to the API, breaking backwards compatibility will not be repeated in future releases of Chisel.

Unlike Verilog, Chisel is sequentially compiled which makes the order in which signals and literals are defined, assigned and referenced critical. References to wires which have not yet been assigned a value up to the current line in the source code will result in an elaboration error. Whilst Verilog permits partial assignment of vectors by default, Chisel supports this operation on vectors(Vecs) exclusively. Scala indexed sequence processing must be performed using methods such as .slice, .zip and .map to perform operations on Vecs before type casting the result back to a UInt to produce a vector [x:y] port in the generated Verilog, which is required where the signal being operated on is a top-level output port. Whilst operations of this nature are possible, such a common hardware operation ought to be more simplistic to perform in Chisel.

Chisel's lack of support for asynchronously reset register forces designers down the path of using synchronous resets 
and prevents them from implementing reset synchronizers within Chisel which is required to generate a clean reset source for the Chisel generated design. Coupled with Chisel's lack of support for multiple clock domains, designers are forced to create a HDL wrapper around the Chisel generated Verilog source in order for the design to be implemented in the design flow.

\section{CONCLUSION}

Chisel has the ability to provide significant productivity benefits to hardware designers where digital designs require repetition as design resources can be focused on the architecture and configurability of the RTL design without concern for the underlying implementation of the HDL generator. Whilst the learning curve to getting moderately complex hardware designs up and running with Chisel is steep, as an understanding of object-oriented programming techniques, Scala, the underlying language that Chisel is implemented with, and Chisel itself are required, configurable hardware components can be realized with a considerable reduction in source code, in a single source language. Although Chisel designers require a foundation in software design to allow them to utilize Chisel's power, constructing circuits with Chisel requires an identical appreciation of the hardware being described to that held by Verilog designers. As designs increase in scale and complexity, and spread across multiple developers, Chisel's object-oriented architecture reduces the amount of repetition required to express complex design, whilst producing a structured and maintainable solution.

While SystemVerilog can provide significant productivity increases over designing with Verilog, the availability to expand on the Chisel API using the constructs and syntax of Scala makes Chisel the perfect choice for performing complex, scalable hardware design such as SoC processor and processor sub-subsystem design. Chisel's lightweight documentation provides sufficient detail to allow designers to get off the ground with Chisel. As the community of Chisel Developers grows, more examples will become available to aid Developers in seeing the advantages of Chisel in practice.

\section{REFERENCES}

[1] A. Waterman et al., "Chisel: Constructing Hardware in a Scala Embedded Language", 1st ed. in Design Automation Conference, San Francisco, CA, 2012. 2

[2] M. Webber, "Arbiters: Design Ideas and Coding Styles", 1st ed. in Synopsys Users Group, Boston, 2001. 3

[3] F. Heilmann et al., "White Paper - Investigate the high-level HDL Chisel", 1st ed. Kaiserslautern, Germany, 2013. 